\title{
Chlorhexidine varnish may be useful adjunct to routine periodontal therapy?
}

\author{
Does chlorhexidine varnish reduce plaque accumulation, gingival index and \\ bleeding levels in people who have chronic gingivitis?
}

\author{
Puig Silla M, Montiel Company JM, Almerich Silla JM. \\ Use of chlorhexidine varnishes in preventing and treating \\ periodontal disease. A review of the literature. Med Oral Patol Oral \\ Cir Bucal 2008; 13:E257-E260.
}

Data sources PubMed, Embase and the Cochrane Library were searched for relevant studies.

Study selection Only controlled human trials of chlorhexidine varnish using clinical outcomes were included: other chlorhexidine preparations (mouth rinses/gels) were excluded, as were studies with concomitant preventive measures, eg, fluoride varnishes.

Data extraction and synthesis Details of data extraction methods are not provided and a qualitative summary is presented.

Results Ten studies were identified. Comparisons were difficult because of considerable variation in the study parameters, such as varnishes with different chlorhexidine concentrations, different ages of participants, number of applications and clinical indices employed. Eight of the 10 studies found improvements in clinical parameters.

Conclusions The application of chlorhexidine varnish seems to have beneficial effects in people who have chronic gingivitis, improving their plaque accumulation and bleeding levels and reducing their gingival index. It is possible to maintain this beneficial effect for prolonged periods of time, although this requires re-applications of the varnish.

\section{Commentary}

The loss of teeth through periodontal diseases affects a significant proportion of the population in many countries. Prevalence studies indicate that between 5 and $20 \%$ of adults display a clinical level of disease activity. Cause-related therapy remains the mainstay of treatment, namely selfdirected plaque control by the patient and biofilm disruption by the professional via scaling and root planing/ surface instrumentation. Complementary therapies or adjuncts to the nonsurgical management of periodontal diseases are also available to the practitioner, taking the form of an antimicrobial or a host-modulating substance. This review describes the evidence surrounding one type of adjunct, the application of chlorhexidine-containing varnish.

The authors describe the literature obtained by undertaking a search of three electronic databases. The process described takes a systematic approach but is not reported in a recognised format for systematic reviews, such as that proposed by the Cochrane Collaboration. The authors identified 36 articles related to the review topic of chlorhexidine varnish and periodontal disease. A review of the abstracts yielded a sample of 11 papers that were selected for full review; one was then rejected leaving 10 papers for inclusion. These papers related to the evaluation of four different varnishes undertaken by seven investigating groups.

The authors provide a descriptive summary of the findings with tabulated summary data. The effect of chlorhexidine varnish application upon pocket depth was described in four studies and the effect upon plaque and gingivitis in six studies. The period of observation in the studies was between 3 and 9 months.

Overall, there was a tendency to see a larger reduction in plaque and gingivitis levels for groups receiving chlorhexidine varnish compared with control groups. With regard to measures of periodontitis (pocket depths), there also appeared to be an advantage in favour of people receiving the varnish. The observed effect was greatest in deeper pockets $(=7 \mathrm{~mm})$ with differences in pocket reduction estimated to be between 0.62 and $1.37 \mathrm{~mm}$ in favour of the groups using the varnish. Two of the 10 studies did not detect a difference between groups for the parameters measured.

Taking a cautious line, not all of the periodontal measures recorded in the studies were reported in this paper, specifically clinical attachment level (CAL), which describes gains in attachment. Therefore, a measure of longitudinal change from a fixed point was not reported, although it was present in the original papers cited. Secondly, the majority of evidence available was from one investigating group. Their data did reveal conflict when considering the long-term effect upon CAL, with one study not showing a difference after 6 months of observation. This then leads to a question surrounding the longevity of the effect from this type of therapy, as the data in these studies were collected over a period of not more than 9 months of observation.

\section{Practice point}

There is some evidence supporting the use of chlorhexidine varnishes as an adjunct to routine periodontal therapy, with greatest impact at sites of more advanced disease. This must be balanced, however, against the prerequisite that conventional periodontal management must meet the highest standards in the first place, and that the long-term impact of this adjunct remains uncertain.

\section{Giles McCracken \\ School of Dental Sciences, Newcastle University, Newcastle upon Tyne, UK}

Evidence-Based Dentistry (2008) 9, 76. doi:10.1038/sj.ebd.6400595 